\title{
Contribution à l'étude des autels sacrificiels du Nya chez les Minyanka du Mali
}

Contribution to a study of the Nya sacrificial altars among the Minyanka in Mali

Philippe Jespers

\section{OpenEdition}

\section{Journals}

Édition électronique

URL : http://journals.openedition.org/span/323

DOI : 10.4000/span.323

ISSN : 2268-1558

Éditeur

École pratique des hautes études. Sciences humaines

\section{Édition imprimée}

Date de publication : 1 septembre 1976

Pagination : 111-139

ISSN : 0294-7080

\section{Référence électronique}

Philippe Jespers, «Contribution à l'étude des autels sacrificiels du Nya chez les Minyanka du Mali » Systèmes de pensée en Afrique noire [En ligne], 2 | 1976, mis en ligne le 04 juin 2013, consulté le 19 avril 2019. URL : http://journals.openedition.org/span/323 ; DOI : 10.4000/span.323 


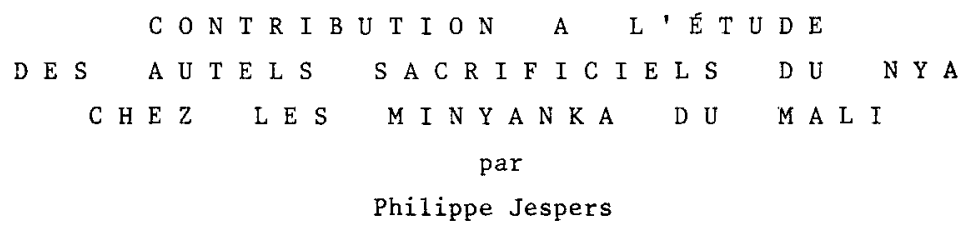

I. Introduction.

Dans la société d'initiation Nya, comme dans la plupart des grandes institutions socio-religieuses du Mandé (Komo, Kworè, Nama), il existe un grand nombre d'autels portatifs qui sont considérés comme les réceptacles des puissances intermédiaires entre Dieu et les hommes. Nous allons essayer de montrer dans cet article que le rôle jouê par ces autels constitue un élëment essentiel de la structure du sacrifice et qu'il permet d'en êclaircir certains des aspects les plus cachés.

Ces autels, thêoriquement au nombre de 266 et répartis dans des sacs, sortent à l'oecasion de deux grandes manifestations annuelies dites nya ka chin (sacrifice du Nya) au cours desquelles ils sont revivifiés par le sang sacrificiel des victimes. La première de ces manifestations se tient en avril-mai, un peu avant les semailles, la seconde en octobre-novembre, après les récoltes.

Ces rites, longs et compliqués, sont effectués en secret dans un enclos de brousse dit nya tun (litt. fourré du Nya), situés à quelques lieues du village. Les autels (yapèrè) y reçoivent pendant le jour le sang des sacrifices propitiatoires effectués par le mufo ("maitre du couteau") au nom des habitants du village et au nom de sacrifiants venus parfois de villages très éloignés du lieu du culte.

Soulignons que ces sacrifices ne peuvent avoir lieu que si les yapèrè (autels) ont été vêhiculës par les "chevaux du Nya" ( les possêdés) jusqu'à l'enclos de brousse. Autrement dit, sans la "prise du possédé" par le Nya - pour parler comme les Minyanka - les yapèrè 
restent obstinément dans la case du village et aucun sacrifice ne peut être effectué dans le nya tun. La résistance du Nya à se manifester est alors interprétée de diverses façons.

Les possédés pénètrent dans l'enclos à reculons, déposent $1 \mathrm{a}$ charge des trois sacs à $1^{\prime}$ 'est en face de trois grandes jarres disposêes à 1 'ouest, divisant ainsi l'espace selon trois axes parallèles orientés vers 1 'ouest. Cette disposition s'explique par le fait que, pour les Minyanka, l'ensemble du cosmos est orientê d'est en ouest (les choses ont été créées à 1 'est). L'enclos du Nya est alors traité, avec les trois sacs, comme un espace défini sur le plan symbolique de la création divine. Les rites s'y déroulent selon le schêma suivant :

(a) transfert des yapèrè des trois sacs dans trois jarres,

(b) égorgement des victimes et libation du sang sur les yapèrè,

(c) consommation des victimes.

Nous allons essayer de montrer que 1 'agencement de ces rites, qui peuvent paraitre oiseux quand on les examine superficiellement et du dehors, se fonde sur la nécessité de ranger les yapèrè dans l'aire sacrificielle en leur assignant à chacun une place au sein d'une jarre.

Disons que cette mise en place des yapèrè se fonde elle-même sur un savoir théorique solidement charpenté (nombres, signes graphiques et devises), comparable d'un point de vue formel à ce que l'on trouve aux fondements des grandes sociétés d'initiation bambara (Komo, Kworè, Nama).

Loin d'être traités par masse indifférenciée - ce dont ils ont pourtant 1 'aspect - les yopèrè sont rangés dans la jarre selon un ordre qu'il nous faut indiquer si nous voulons comprendre leur position dans 1 'aire sacrificielle qui est ici microcosme de 1 'univers.

Nous croyons même qu'il est nécessaire de distinguer un dispositif symbolique qui est d'abord mise en place des yoxpèrè, opération logique de classement des forces de l'univers, de l'utilisation sacrificielle qui peut en être faite à des fins diverses. C'est pourquoi $j$ 'esquisse ici quelques êléments d'une connaissance initiatique qui constituent un minimum pour comprendre l'ordre de disposition des yapèrè dans le nya tun au moment des grands sacrifices annuels. 
Contribution à l'étude des autels sacrificiels du Nya

II. Nombres.

Selon une très vieille tradition, les autels du Nya se répartissent dans trois sacs de toile de coton d'inégale grandeur : un grand, un moyen, un petit. Chaque sac est destiné à recueillir une même série de 85 yapèrè, à l'exception toutefois du petit sac qui en recueille "un de plus", soit :

(a) 85 yapèrè dans le "sac mère", bugrqmu, dit aussi "sac de 1'âme du ciel et de la terre" (grand sac).

(b) 85 yapèrè dans le "sac du milieu", bwgroya, dit aussi "sac de 1 'âme des personnes et des animaux" (sac moyen).

(c) $85+1$ yapèrè dans le "petit sac", bugropirè, dit aussi "sac de I'âme de toutes choses" (1). Soit 256 yapèrè. A cela s'ajoute, gravitant dans l'orbite des sacs et des jarres, le bâton du Nya (manipulé par le possédé), l'anneau du nankon (de la société des chasseurs), attaché à la "bouche du sac", 1a clochette du Nya, suspendue sur la ventrée du sac, les couteaux sacrificiels utilisés pour l'égorgement des victimes, des calebasses destinées à recueillir le trop-plein du sang... Tous ces yapèrè sont également transférés dans le nya tun au moment des grands sacrifices annuels.

I1 est clair qu'en suivant les principes de cette répartition, on aboutit au compte de 266 éléments qui est le nombre de base de la création mandé. Soit $3 \times 85+1=256+10=266$. S'applique ici comme chez les Bambara la devise énoncée par le vieil initié du Komo : "le mystère de la création est dans les nombres".

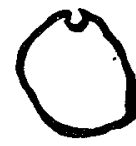

85 yapèrè "sac mère" ciel-terre

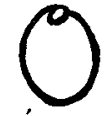

85 yapèrè "sac du milieu" homme-animal

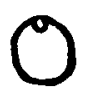

$85+1$ yapèrè "petit sac" choses

(1) Nous prenons ici en considēration un nya mouso (Nya ferme). Il existe par ailleurs un nya $k a$ mo (Nya au bras gauche qui est fait de quatre sacs). 
Il convient toutefois de souligner que dans cet ensemble à 266 éléments des regroupements sont opérés selon un système de correspondance numérique qui semble spécifique au Nya.

La force de ce modèle tient à sa cohérence : les trois sacs contiennent chacun la même série de 85 yapèrè, avec un yapèrè de plus dans 1e "petit sac". Ceci implique qu'au moment de leur constitution, les yapèrè sont reproduits trois fois et répartis dans trois sacs. Au moment du sacrifice, ils sont disposês en trois jarres, à 1 'ouest du nya tun. Pour 1'initiê, cela signifie que chaque yapèrè s'implique dans 1 'espace de la création du "ciel et de la terre" (nom du grand sac), dans celui de la création des "hommes et des animaux" (nom du sac moyen) et dans celui de la création des "choses" (nom du petit $\mathrm{sac)}$. Les 85 yapèrè sont ainsi reproduits trois fois selon un mode de classement de type génétique qui concerne également 1'univers, 1 'humanité et les choses. Soit trois niveaux où le yapèrè peut également exercer une fonction majeure (assurer 1a protection des 1ignages, favoriser la procréation, faire venir la pluie, etc...).

Cette triple implication du yapèrè trouve cependant sa propre limite dans la mesure où le "petit sac" recèle un yapèrè de plus que les deux autres. Ce yopèrè doit alors retenir toute notre attention. Au cours d'une récente enquête, j'ai été confronté au problème de ce yapèrè en position à part dans le "petit sac" du Nya, sans avoir pu résoudre toutes les questions qu'il soulève. Ces questions touchent à la fois à sa nature, à sa position par rapport aux autres yapèrè et à sa manipulation au moment des rites sacrificiels (par exemple, il est interdit d'y verser du sang). Mais essayons d'abord de définir la nature de ce yopèrè qui joue, nous le verrons, un rôle considérable dans le Nya. Tous les initiés s'accordent à reconnâ̂tre qu'il est 1 'habitacle de la puissance de "génies" ancestraux exceptionnels, les djine blew ("génies rouges"), dits sefè (ancêtres). Le terme sefè, qui est ici le nom du yapèrè, s'applique généralement aux esprits ancestraux et désigne ici plus particulièrement les premiers jumeaux de tyélèrè, "1a vieille petite femne", bien connue chez les Bambara sous le nom de Mousso Koroni.

Les Minyanka dêcrivent ces "ancêtres" comme des jumeaux mixtes nés avec un retard considérable (12 mois de gestation) (2), soudés un à un à un tronc unique et le corps tuméfié par la lèpre. Le jumeau se 
Contribution à l'étude des autels sacrificiels du Nya

nomme nanya (1itt. homme venu) et la jumelle pamanygh (1itt. qui est bien venue), noms que 1 'on donne chez les Minyanka aux enfants nês

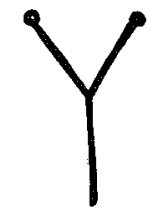

signe des sefè

(ancêtres) après terme (naissance perçue comme bénëfique). Ces jumeaux, nés lépreux pour une intelligence supérieure (3), se sont imposés comme les génies tutélaires du village (ou propriétaires originaires de la terre). A ce titre, ils habitent le "bois sacré" dit kachin kan, situé aux abords du village, où la communauté leur offre annuellement un sacrifice (généralement un taureau). Mais, si le bois sacré est habituellement leur siège, ils peuvent brusquement changer de lieu et pénëtrer dans le "petit sac" du Nya où ils trouvent leur support. Ils mettent alors la selle sur un "cheval" (un possêdé) et rentrent au village pour y imposer leurs prérogatives à titre de propriêtaires du village et du Nya en même temps.

Ces crêatures de brousse, nés d'une gestation excessive, sont par ailleurs censês jouer un rôle décisif dans tous les rites liês à la fêconditê.

Le yapèrè qui leur sert de support dans le. "petit sac" du Nya est fait d'une dêfense de phacochère et de deux morceaux d'or, symbole de l'intangibilité divine qui les anime, le.tout étant solidement enveloppé d'une boule de coton rouge.

Mais, si les jumeaux peuvent brusquement pénétrer dans I'habitacle du yapèrè, celui-ci peut également être manipulê, voire même excité, par le chef de la société du Nya, le nya $f \varrho$, en vue de provoquer une possession dite "prise du cheval par le Nya". Le nya fo rentre alors dans la case du Nya, se penche sur le yapèrè posé en haut du sac et énonce la formule suivante :

fizi, fizi, fizi, Kotigi, fizi

prends, prends, prends, Kotigi, prends (ou fais tomber)

(2) Un mythe raconte comment les yapèrè du Nya étaient à l'origine contenus dans des placentas d'antilopes ayant peiné à mettre bas.

(3) La lèpre est perçue ici comme une déficience qualifiante. On prête au lépreux une intelligence supérieure (plus ou moins teintée de ruse). D'autre part, on dit que le Nya prend parfois l'aspect d'un lépreux pour se manifester à un homme. 
nanya, $i$ tyon $i$ tok byan

Nanya (jumeau), si tu es absent, ton nom est $1 a ̀$

fili, fizi, fizi, kotigi, fizi

prends, prends, prends, Kotigi, prends

pamanygh $i$ tya, $i$ to $k_{Q}$ byan

Pamanyoh (jumelle), si tu es absente, ton nom est là.

Cette invocation à tonalité magique laisse entendre que le yopèrè est le réceptacle des jumeaux nanya (le mâle) et pamany̧h (la femelle) à qui l'officiant attribue tour à tour le titre de kotigi, "propriétaire de la chose du Nya". Cette formule est également énoncée au temps de la confection de tous les yapèrè, c'est-à-dire au temps même de 1 a constitution du Nya. Tout ceci donne au yopèrè du "petit sac", dit "sac de l'âme de toutes les choses", un statut exceptionnel et justifie aux yeux des initiés sa position à part, an kè̉è, dans le Nya.

Nous savons par ailleurs que, dans la numérologie bambara, la position à part d'un objet dans une aire cultuelle est symbole de 1 'unicitê et de la singularité de l'être qu'il représente. De même que le nombre 1 (4).

III. Signes graphiques.

L'enquête a révélé, comme chez les Bambara, l'existence d'une planchette des signes dite nya ti wala (planchette des signes du Nya), qui constitue, comme au Komo, "1'un des biens intellectuels les plus précieux de la sociêté" (5). Taillêe dans le bois du fromager (banco $k g r g)$, de forme rectangulaire, elle repose dans la case du Nya sur une petite banquette, orientée vers 1 'ouest (à l'abri des regards). Elle comprend une sërie de 86 signes graphiques - ou signes fondamentaux du Nya - considérês comme étant issus de la pensêe de ǩ̉è (1e Dieu créateur).

La genèse des signes exprime le tracé de la création divine, la

(4) Germaine Dieterlen et Youssouf Cissé, Les fondements de la société d'initiation du Komo, Paris-La Haye, Mouton, 1972, p. 76.

(5) Ibid., p. 64. 
Contribution à 1 'étude des autels sacrificiels du Nya

formation du ciel et de la terre, la formation des "premiers ancêtres", la descente du Calao sur terre, etc... Et contrairement, semble-t-il, à la série du Komo (6), celle du Nya suit, du moins en ce qui concerne les premières graphies, 1'ordre de succession des événements mythiques que nous connaissons. C'est ainsi que le signe affecté aux "premiers ancêtres" (sefèlè) est en tête de cette série et celui affectê aux ancêtres de la deuxième génération (sefè) est en deuxième position.

Cette genèse des signes nous plonge dans le mystère le plus reculé de la formation des yapèrè, car, pour les Minyanka, les signes du Nya, manifestés dans la pensée de $k$ lè, ont précédé 1 a création des yapèrè qu'ils désignent. Les 86 signes graphiques de la planchette correspondent en effet un à un aux 86 yopèrè du "petit sac" du Nya. C'est pourquoi, au temps de leur constitution en brousse, tous les yapèrè sont soumis à une épreuve de contact avec les signes qui leur correspondent sur la planchette et sont ensuite proclamés grâce à leurs devises (signes et devises dont nous avons la nomenclature). Ce rite, effectué pour chaque yapèrè par le kara mokg, mâ̂tre du kara (cercle), assure dans l'enclos de brousse une sorte de transmutation de l'essence du signe en l'être même du yapèrè. Cela revient à dire que les signes du Nya, manifestations de la pensée de klè, préexistent aux yapèrè et les font venir à l'existence.

Les yapèrè sont ensuite reproduits trois fois avant d'être répartis dans trois sacs de façon à aboutir, sur 1 a base de cette multiplication, au compte de $256(+10)$, nombre de base de la création au Mandé. Les 266 yapèrè sont alors dans les sacs les témoins des signes de la création de klè ; ils garantiront sa durée et sa perpétuelle vitalité au village, assurés et augmentés par des sacrifices sanglants effectués dans 1 'enclos de brousse. On clame à leur sortie :

"Antilope, ta chose est semblable à la chose de klè." Cette image de 1 'antilope a des implications beaucoup plus vastes qu'on ne peut le supposer d'abord et conditionne une représentation liée à l'origine des sacs. Car, ici comme dans la plupart des grandes sociêtês d'initiation bambara, les sacs représentent symboliquement le placenta divin dont résulte toute la création de l'univers. Les

(6) Ibid., p. 79 


\section{Philippe Jespers}

sacs sont dits kongonygh, "commencement du monde", ou klè sgara, "placerita de klè" ou, comme l'indique le nom du premier sac, bworomu, "sac mère". Les Minyanka ont cependant une façon originale d'expliquer 1 'origine des sacs du Nya. Ils racontent comment les warablew (singes rouges), anciens propriétaires du Nya en brousse, furent amenés par le Calao à recueillir les yopèrè dans des placentas d'antilopes ayant eu toutes les peines à mettre bas. Or il va de soi que dans la pensêe du mythe le placenta de 1 'antilope reprêsente celui des jumeaux du Nya nés avec un retard considérable (voir p, 130). Et cette déficience est, nous le savons, qualifiante aux yeux des Minyanka.

IV. Rite sacrificiel.

A. Transfert des yapèrè dans la première et la deuxième jarre sacrificielze.

Les possédés ayant déposé le fardeau des trois sacs dans le nya tun, les rites sacrificiels vont pouvoir être effectués par le mifo ("mâttre du couteau").

La première partie de ce rite, qui est de loin la plus complexe, consiste à transférer dans les jarres les yapèrè contenus dans les sacs. Le rite exige un déplacement des yapèrè d'est en ouest. Ceci s'explique par le fait que, pour les Minyanka, l'ensemble du cosmos est orienté selon un axe qui va de l'est vers $1^{\prime}$ 'ouest.

Faisant donc face à 1 'ouest, le sacrificateur s'accroupit devant 1 a jarre et tournant ostensiblement le dos au "sac mère", il tâte de la main gauche (7) la "bouche du sac" afin d'en extraire un premier yapèrè. Après une brève manipulation impliquant un pouvoir de reconnaissance tactile des yapèrè contenus dans le sac, il en sort un qui a la forme d'une grosse boule brunâtre. Il le dêpose à terre tout en murmurant la formule suivante :

sefèzè ye kafali ye koolo

premiers ancêtres pardon, voici votre vieux chemin

(7) La gauche est symbole, chez les Minyanka, de la masculinité et de la" virilité. 
Contribution à l'étude des autels sacrificiels du Nya

Cette prière doit être énoncée à voix très basse de façon à ne pas divulguer. le nom des "premiers ancêtres". Pour l'initié, il n'en demeure pas moins que ce yapèrè est en vêrité celui dit sefèzè, "les premiers ancêtres".

La prohibition de clamer le nom des ancêtres apparaît comme une propriété structurale d'un certain type de dênomination rituelle que 1 'on retrouve dans de nombreuses sociétés d'initiation au Mandé. Un processus analogue est à I'oeuvre, nous semble-t-il, au moment des sacrifices annuels aux autels du Komo. Le sacrificateur, en traçant sur le sol des signes, récite à haute voix leurs noms tandis que les sociétaires "se bouchent les oreilles et ferment les yeux pour ne pas, disent-ils, entendre, voir, et partant comprendre la genèse de l'univers" (8). On le voit, le rite n'a de sens ici que placé dans un rapport avec le registre de la création divine. En sortant les sefèzè du "sac mère", le sacrificateur du Nya accomplit un geste qui rappelle 1 'oeuvre de création des premiers ancêtres (kzè les ayant fait descendre dans le monde en les enveloppant dans un grand sac transparent).

Le yapèrè dit sefèlè (terme appliqué généralement aux esprits ancestraux) est constituê d'une dêfense de phacochère contenant un grain d'or, représentant nyglèxè (litt. vieux petit commencement) et d'une racine de caillcédrat représentant tyètèrè (1itt. vieille petite femme). Les deux éléments sont enroulés dans une boule de coton rouge ètroitement serrée par des liens.

Ce yopèrè correspond atı premier signe de la planchette, dont il

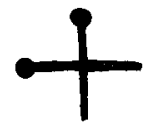

signe des sefèze

"premiers ancêtres" est issu. Le signe est une croix grecque figurant 1 'union des "premiers ancêtres", tyèzèrè, la mère-terre orientée nord-sud, et nyglèrè, le père-ciel orienté est-ouest. Les droites se recoupent en un point qui se dit tyèrè tipize, "tronc des petites personnes", ou tyèrè kzè ni ningè, "tronc du ciel et de la terre", le point d'intexsection exprimant ici un rapport commun de la structure du monde (ciel-terre) et

(8) Germaine Dieterlen et Youssouf Cissé, Les fondements de la société d'initiation du Komo, Paris-la Haye, Mouton, 1972, p. 80. 
de la structure de 1 'humanité (les ancêtres) (9).

Créatures célestes descendues sur terre après une querelle au ciel, les sefèlè ont une relation privilégiēe avec les âmes (muna) au ciel. Aussi n'est-il pas étonnant que le signe des sefèzè soit uti1isé dans de nombreux rituels liés à la fécondité. Il est tracé plusieurs fois à la crème de petit mil sur le corps des nouveaux-nés (sur le dos, le front, la poitrine, les mains, la bouche), le jour de leur consécration au Nya (ou à toute autre puissance). Car on dit que le Nya a favorisé leur naissance. Une des fonctions du yapèrè des sefèzè consiste en effet à coopérer le long du "fromager" à la descente des âmes (muna), initialement conçues au ciel.

Revenons à présent à la trame du rituel. Le transfert des youèrè du' "sac mère" à la jarre sacrificielle se décompose en trois séquences bien distinctes, selon un axe qui va de l'est (le sac) vers l'ouest (1a jarre).

Séquence I. Le "sac mère" étant posé derrière lui (à 1'est), le sacrificateur en extrait d'abord le yapèrè des sefèzè ("premiers ancêtres"), puis il le dépose au pied de la jarre. Cette disposition implique qu'il lui est absolument interdit de le voir sortir du sac.

Séquence II. A peine $a-t-i l$ extrait ce premier yapèrè qu'il saisit le "sac mère" qu'il maintenait jusque-1à par devers lui et 1 'intercale entre ses jambes et la jarre. Ainsi, avant de sortir tous les autres yapèrè, change-t-i1 sa propre position par rapport au "sac mère" qu'il va maintenant manipuler de face. C'est ainsi qu'il transfère un à un tous les yapèrè dans la jarre, tandis qu'il maintient toujours les "premiers ancêtres" à terre.

Séquence III. Le sac une fois vidé, il reprend le yapèrè des sefèzè, laissè à terre, et le pose en haut de la jarre. Les "premiers ancêtres" coiffent alors tous les autres yapèrè et, se servant d'eux comme d'un socle massif, fait de 84 yapèrè de toutes formes, ils dominent la jarre sacrificielle.

- . Telle est la règle de ce "mouvement objectif apparent". En sortant les sefèzè du"sac mère" avant tous les autres, en les maintenant d'abord à terre pour les transfêrer ensuite en haut de la jarre, le

(9) Dans de nombreux rẻcits mythiques, les premiers ancêtres de 1 'humantité sont identifiés au couple ciel-terre. 
Contribution à l'êtude des autels sacrificiels du Nya

sacrificateur suit un ordre qui implique, croyons-nous, deux mouvements bien distincts.

-- Le premier suit un ordre dont on peut supposer que le principe est celui-même qui a présidé à la naissance des ancêtres sortant les premiers du "sac du monde", kongony̧̧h, (mémoire bio-filiative).

-- Le second suit un ordre dont on peut supposer qu'il est hiërarchique : les ancêtres occupent le haut de la jarre en tant que médiateurs entre le ciel et la terre. Leur position signifie l'association concomitante de leur rôle avec les forces de 1 'univers (cielterre) et l'utilisation de ces forces par les communautés. Et c'est ce qui explique, croyons-nous, l'attitude de soumission du sacrificateur au moment où il dëpose le ycoèrè à terre en murmurant : "pardon, premiers ancêtres, voici votre vieux chemin".

Danielle Jonckers a bien su montrer dans 1'étude qu'elle consacre au sacrifice chez les Minyanka, comment cette attitude de soumission devant 1e Nya, ou toute autre puissance, est au fondement de "l'ordre social" dont les yapèrè sont à bien des égards les garants (10).

Mais la portée de ce rite de transfert apparâ̂t plus clairement si on le reporte au système graphique dont nous avons déjà parlé plus haut. Il est en effet aisé de montrer que la position de chaque yapèrè dans la jarre est en affinité avec la position du signe qui lui correspond sur la planchette. Selon les informations recueillies auprès des initiés, les yapèrè sont rangês sous l'action signifiante de la structure graphique de la planchette du Nya. En effet, la position du yapèrè des "premiers ancêtres" en haut de la jarre renvoie à la position du signe qui lui correspond en tête de la série graphique. De même, la position des yapèrè d'Orion et de Sirius, qui forment un socle sur lequel reposent les "premiers ancêtres", renvoie au troisième et quatrième signe de la planchette. Tout porte à croire que le sacrificateur dispose ici d'une mémoire qui lui permet de rétablir un ordre de classement des yapèrè sinon identique à l'ordre des signes, du moins de même type. Référons-nous à cet égard à la première série

(10) J'emprunte ici l'expression d"ordre social" à Danielle Jonckers qui a montré comment les yapèrè sont des liants de l'ordre social et des facteurs signifiants de raffermissement de la structure lignagère. Cf. Contribution à l'étude du sacrifice chez les Minyanka. 
des signes graphiques du Nya (à lire de gauche à droite) (11).

signes

yapèrè

$$
\begin{array}{cc}
\text { sefèzè } & \text { sefè } \\
\text { "premiers } & \text { "ancêtres" } \\
\text { ancêtres" } & \\
1^{\circ} \text { génération } \quad 2^{\circ} \text { génération }
\end{array}
$$

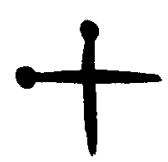

1

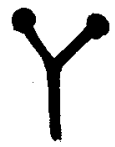

2

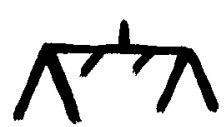

3

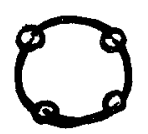

4

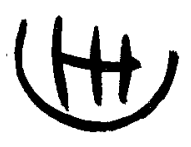

5 wolotipya yèngènyè cho

"homme

étoile"

Orion

$$
\text { "co-épouse }
$$$$
\text { de la lune" }
$$

Sirius klè tonyungo

"tête du calao"

Calao

\section{fonctions}

$$
\begin{gathered}
\text { propriétaires originaires } \\
\text { de la terre }
\end{gathered}
$$

fécondité de

la terre et des femmes piuie

J'indique ici l'ordre de disposition, dans le haut de la jarre, des youèrè correspondant un à un aux premiers signes de la planchette. Ce sont dans l'ordre de leur transfert :

-- klèto nyungo, la tête du Calao (signe 5)

Posée au nord de la jarre, parfaitement reconnaissable avec son long bec, nouée de fil de coton, la tête du Calao est, comme l'indique sa devise, un instrument magique d'action sur la pluie.

na klè ni sanh wo tebe pe yapèrè ni

toi qui au nom de klè fait venir la pluie dans les yapèrè de nos ancêtres

Selon un mythe, c'est le jour de la mort de sa mère que le calao fut investi de son pouvoir de faire la pluie. Ce jour-1à, la terre était tellement sèche qu'il ne put creuser une tombe pour elle. Alors il invoqua klè pour lui demander la pluie et la pluie vint ramollir la

(11) Cette présentation n'implique pas de notre part l'affirmation que la planchette est réellement utilisêe pendant les rites sacrificiels au nya tun. Elle reste à l'abri des regards dans la case du Nya, posée sur une banquette orientée vers 1 'ouest. Il n'en demeure pas moins qu'elle est un système graphique qui garantit l'intelligibilité du rangement des yapèrè dans la jarre. En outre, elle peut correspondre à un ordre d'explication adopté par les initiés. Pour chaque yapèrè, mes informateurs traçaient sans hésiter un signe sur le sol et énonçaient la devise du signe ou du yapèrè. Enfin, rappelons que la planchette est réellement manipulée au temps de 1a constitution d'un Nya : chaque yapèrè est alors soumis à une épreuve de contact avec le signe qui lui correspond et proclamé grâce à sa devise. 
Contribution à l'étude des autels sacrificiels du Nya

terre ; il put ainsi creuser une tombe pour sa mère (12).

-- yèngènyècho, la "co-êpouse de la lune" (signe 4)

Ce yapèrè, fait d'un conglomérat de huit racines, est posé au centre de la jarre et désigne Sirius qui, dans la cosmologie minyanka, est la "co-épouse de la lune". Yèngènyèchg témoigne dans le ciel de la métamorphose de pamanygh (litt. qui est bien venue), première fille de tyèzèrè, en corps lunaire. Le yapèrè est utilisé come un instrument magique d'action sur la fêcondité des femmes. Voici sa devise :

nyi nyi klè ni sikan fè chys

terre calme, toi qui au nom de klè enraye la jalousie des femmes sorcières

-- wolgtipya, "l'homme-ëtoile" (signe 3)

Wolqtipya désigne la constellation d'Orion. Le yapèrì̀ est fait d'un conglomérat de huit racines et d'une pierre de foudre. I1 est posé au centre de la jarre à côté du yapèrè de yèngènyècho, la "coépouse de 1a lune". L'appariement des deux étoiles s'explique en fonction d'un code cosmologique. En effet, si yèngènyèch̆g est le témoin de la métamorphose de pamanygh (signe 2) en corps lunaire, conjointement wolqtipya tëmoigne de celle de nanya (litt. homme venu), premier fils de tyèzèrè (la "vieille petite femme"), en corps solaire. Le mythe raconte qu'après s'être affrontés en un combat singulier dont une tortue de terre avait été l'enjeu, nanya projeta pamany̧h à terre. Au terme du duel, nanya se transforma en soleil et pamonygh en lune.

Créatures nées de tyèzèrè, nanya et pamanygh sont ainsi liés à la fécondité du soleil et de la lune, et c'est wolotipya (Orion) et yèngènyècho (Sirius) qui en témoignent dans le ciel. Eloquente à cet égard est la devise du yapèrè d'orion :

ma dyo kiè ni sefe ni worg chyg

toi qui parle au nom de klè (Dieu créateur) des sefè (ancêtres) et de l'étoile qui traverse la nuit

Elle dit en clair que le yapèrè est ici le témoin qui "parle" au non des sefè (nanya et pamany̧h, signe 2), ancêtres de la deuxième génération mythique, et de la constellation qui traverse la nuit, c'est à

(12) Selon un mythe que nous avons enregistré, le calao descendit sur terre avec la planchette des signes. 


\section{Philippe Jespers}

dire Orion comme témoin du soleil. Or, nous avons vu que le yapèrè des sefè est le seul des 86 yapèrè à être absent des deux premières jarres (il n'est présent que dans la troisième). I1 s'ensuit dès lors qu'Orion est, comme le dit la devise, le témoin du soleil absent, mais absent aussi sous 1a forme de son yapèrè dans les jarres 1 et 2. La spécificité de sa position s'explique au travers du dispositif de rangement des 266 uapèrè.

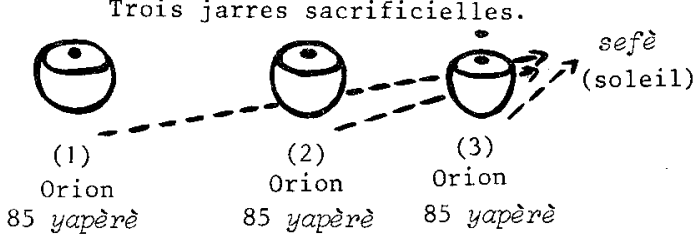

Mais elle s'éclaire aussi si nous nous reportons au code cosmogonique. Orion est en effet la constellation qui, pendant la saison chaude, traverse la nuit (wgrg chyg) et qui disparât à l'occident. A ce titre, les Minyanka le décrivent comme un soleil nocturne traversant le ciel d'est en ouest. Un peu avant les semailles (mai-juin), sa disparition est interprétée comme une pênétration fécondante du soleil en terre.

Des rites nocturnes sont alors effectués sous le signe de sa course. Un peu avant les semailles, durant la nuit de la grande fête du Nya (lors d'un rite appelé "réfection annuelle du Nya"), les initiés mettent le feu au toit de la case du Nya. Mais c'est Orion, disent-ils, qui consomme 1'incendie avec son feu sacrificiel au moment où le soleil est précisêment au nadir.

Tout porte à croire que nous nous trouvons ici en présence d'un code cosmogonique coordonnant la course du soleil (le jour) et la course d'Orion (la nuit) selon un axe qui va du nadir au zénith. Il convient de souligner à cet égard que la position du soleil au nadir en terre - est interprétée par les initiés comme la position fécondante du Nya.

Germaine Dieterlen a pu situer avec ses informateurs bambara 1a position du Nya dans l'ensemble des grandes sociétés initiatiques, selon l'ordre suivant : le Nya est au nadir, le Komo à l'ouest, le Kworè à l'est, le Nama au sud, le Kono au nord, chaque sociêté étant mise en rapport avec l'organisation universelle des espaces et des 
Contribution à l'étude des autels sacrificiels du Nya

\section{éléments (13).}

Il existe également un rite nocturne d'anti-sorcellerie durant lequel Orion exalte dans le ciel la puissance du Nya. Ce rite dit nya tyèré saraka ("offrande d'oeuf de poule au Nya") a pour fonction de prémunir la comnunauté contre l'agression des sorciers. Il est recommandé aux sacrifiants de déposer devant la case du Nya un oeuf de poule dans une petite jarre d'eau et d'attendre la chute d'orion à I'ouest. La chute de la constellation est censée communiquer 1a chaleur au Nya. L'oeuf est alors instantanément bouilli et consommé par les sacrifiants. Les spécialistes affirment que le ycopèrè d'Orion a coopéré dans le Nya à ce bouillonnement de 1 'oeuf (14) au moment de la pénétration d'orion en terre (environ à quatre heures du matin).

-- sefélè, les "premiers ancêtres"

Ayant déposé les yapèrè d'Orion et de Sirius (signes 3 et 4 ) au centre de la jarre, le sacrificateur transfère en dernier lieu le yapèrè des sefèlè (signe 1), les "premiers ancêtres".

C'est ainsi que s'achève le transfert des yapèrè dans les deux premières jarres.

Sur ces bases, et en voulant aborder la question du sacrifice, on voit bien qu'il passe obligatoirement par une classification des forces contenues dans l'univers.

L'ordre de disposition des yapèrè qui contiennent ces forces dans la jarre se présente comme suit :

Zênith

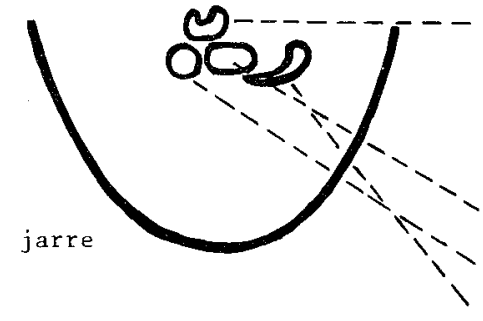

sefe sefèzè

wolotipya orion yèngènyècho Sirius klèto nyungo tête du calao

ancêtres ancêtres signe 2 (soleil-lune)

premiers signe 1

(ciel-terre)

nadir

(13) G. Dieterlen, Essai sur la religion bambara, P.U.F., P. 232

(14) Selon les initiés, 1 'oeuf offert au Nya représente 1'oeuf cosmique initial d'où sont sortis les "premiers ancêtres". 


\section{Philippe Jespers}

Les yapèrè sont disposés selon un axe cosmogonique qui va du nadir (fond de la jarre) au zénith (haut de la jarre).

-- Le yapèrè des sefèlè (signe 1), les "premiers ancêtres" identifiés (par le signe) au couple "ciel-terre", occupe le haut de la jarre en s'appuyant sur le socle formé par les yapèrè d'Orion et de sirius. -- Les yapèrè de wolotipya (Orion) et de yèngènyècho (Sirius) sont appariés au centre de la jarre comme témoins du soleil et de la lune en position nocturne sous le yapèrè ciel-terre qu'ils soutiennent. -- La tête du Calao est posée au nord à côté des yapèrè d'Orion et de Sirius.

Enfin rappelons que le yopèrè des sefè (signe 2), "ancêtres" identifiés au couple soleil-lune, est le seul des 86 yoxpèrè à être exclu de cette jarre. Le dispositif du Nya, impliquant son absence dans les deux premières jarres et sa présence dans la troisième, permet de penser que ce yapèrè, à la fois dehors et dedans, est à la charnière des relations entre le cosmos et la jarre, un des agents les plus puissants de la médiation (15).

La jarre est de ce fait liée à l'harmonie dynamique rigoureuse du cosmos.

Tel est 1 'ordre de disposition des yapèrè occupant le haut de la jarre. Il conviendrait, si la place ne nous manquait, d'examiner tout aussi attentivement la position des autres yapèrè. Disons seulement que la jarre sacrificielle contient des séries distinctes de yapèrè nommées habituellement "chemins" et rangées selon un ordre que nous indiquons ici sommairement (16).

-- Le tyèmèkoro, le "chemin de la connaissance", regroupe au fond de la jarre des yapèrè liês à la divination. Ils correspondent aux derniers signes graphiques de la planchette du Nya et constituent le "fondement de la connaissance".

-- Le kgm koro, le "mauvais chemin", regroupe des yopèrè qui trempent dans la sorcellerie. Les initiês savent que se cachent à ce niveau

(15) Ce yapèrè semble en effet à une position charnière entre la jarre et le monde, car c'est lui qui prend les possédés du Nya ou coopère à leur prise.

(16) Outre leur regroupement par "chemins", les yapèrè sont classés en masculins/féminins, diurnes/nocturnes, bénéfiques/maléfiques. 
Contribution à 1 'étude des autels sacrificiels du Nya

des yapèrè qui leur donnent en certaines circonstances le pouvoir maléfique d'agir sur autrui (mise à mort à distance, etc...). -- Le sike fèn koro, le "chemin des propriétaires de la brousse", regroupe des yapèrè qui sont généralement faits de racines prélevées dans les lieux-dits des djine (génies). Leur fonction est de transmettre au Nya toute information émanant de ces génies ou puissances attachés aux lieux-dits (colline, bois, mare). Ces génies (les sike chyèn : nains aux pieds à l'envers ; les turugbuere : génies sourds ; les tegere : serpent boa ; fari : génie unijambiste de la mare) sont en vérité les propriétaires originaires de la brousse qu'ils se partagent jalousement depuis leurs ruptures respectives avec tyèllèrè, leur mère (17). Leur agression constitue I'un des périls les plus graves qui puissent menacer les communautés. C'est pourquoi on leur offre de nombreux sacrifices (kara) aux lieux-dits.

-- Le fanga koro, le "chemin des cimetières", regroupe des yapèrè qui sont des sortes d'êmissaires pouvant servir de relais dans les rapports entretenus entre le village des vivants et le village des morts (au moment des conflits entre les deux villages).

-- Le chyg koro, le "chemin des jarres", regroupe des yapèrè liés aux jarres de fondation de quartiers de village et de sociêtés d'initiation (autres que 1e Nya).

-- Le Zoozo koro, le "chemin de la chasse", regroupe des yapèrè de chasse.

-- Le muama koro, le "chemin des jumeaux", regroupe en haut de la jarre la constellation des grandes puissances : les yapèrè d'Orion et de Sirius (signes 3 et 4 ), témoins de la puissance de la deuxième génération mythique (signe 2) ; le yapèrè des sefêlè représentant ou contenant la puissance des "premiers ancêtres" (signe 1).

La liste des "chemins" ou signes qu'on vient d'énumérer, montre ce qu'il en est de la jarre au terme de ce long transfert : elle est 1 'expression compacte sous 1 a forme de 86 yapèrè d'une partition complète des forces qui régissent l'univers, mais elle est, en même temps, un vaste répertoire des espaces ou sous-ensembles d'espaces (généalogiques) mis en relation avec ces yapèrè.

(17) Ces génies de lieux figurent sur la planchette des signes du Nya. 
Les initiés peuvent ainsi repérer dans la jarre plusieurs "chemins de yopèrè", ceux d'un même chemin se regroupant par leur fonction commune (assurer la protection des familles, favoriser la descente des âmes, lutter contre la sorcellerie, etc...), par leur rôle joué dans des lieux de séjoux privilégiés (cimetières, collines, mares, etc...) ou encore par la forme de l'objet fabriqué (yapèrè signifie littéralement chose fabriquée).

Dès lors, en observant attentivement ce rite, on ne peut manquer d'être frappé par l'extrême minutie avec laquelle l'officiant effectue la mise en place des yapèrè à laquelle tous les sociétaires accordent du reste une attention soutenue (il arrive même que ceux-ci dẻsapprouvent un officiant qui ne suit pas la règle).

J'ai pu remarquer par ailleurs que ce rite s'effectue dans un débordement de commentaires joyeux ayant trait à toutes sortes de situations vécues mises en relation avec tel ou tel yapèrè. Par exemple, un sociétaire montre du doigt un yopèrè qui a eu une influence favorable sur le cours de sa vie. Un autre manipule un yapèrè en rappelant qu'il lui a été légué par son père, etc...

La force avec laquelle s'effectue 1 'opération logique de classement dépend aussi de 1'intensité avec laquelle le sujet y participe activement.

B. Transfert des yapèrè dans la troisième jarre sacrificiezze.

Nous avons insisté plus haut sur 1'importance qu'il faut accorder à la répartition des yapèrè dans trois sacs. Nous avons vu que ce dispositif marquait un yapèrè en position "à part" dans le "petit sac", ce yapèrè étant le réceptacle de la puissance des sefè (signe 2), "ancêtres" de la deuxième génération mythique et propriétaires mystiques du Nya.

Maintenant, après avoir mis en évidence le statut de ce yapèrè dans un ensemble de 266 éléments, il nous reste à voir comment le sacrificateur va le transférer dans la troisième jarre, de même que les 86 yapèrè du "petit sac".

Ici, comme pour les deux premières jarres, il obéit à un ordre rigoureux en ce sens qu'il suit le "chemin des yapèrè" (de bas en haut, voir p. 126), de sorte que, au terme de cette opération, les 
Contribution à l'étude des autels sacrificiels du Nya

derniers yapèrè transposés dans le haut de la jarre correspondent aux premiers signes graphiques de la planchette : la "tête du Calao" (signe 5), la "co-épouse de la lune" (signe 4), "1'homme-étoile" (signe 3). Restent alors les deux premiers yapèrè correspondant aux deux premiers signes affectés, on $1^{\prime}$ a vu, aux deux premières générations d'ancêtres (signes 1 et 2).

Compte tenu de la position exceptionnelle des sefè (signe 2) dans la généalogie mythique (les sefè sont les jumeaux du Nya), on peut se demander comment le sacrificateur dispose leur yapèrè en haut de la jarre. Obéit-il à l'ordre de succession des signes de la planchette (4-3-2-1) ? A vrai dire non. Il canalise la sortie des yapèrè en modifiant et en inversant 1 'ordre de succession des signes en 4-3-1-2, de telle sorte que le yapèrè des sefè (signe 2), ancêtres de la deuxième génération, coiffe celui des sefètè (signe 1), ancêtres de la première génération. Puis il entoure le yapèrè des sefè (signe 2) de l'anneau du nankon (yapèrè de la société des chasseurs) en murmurant la devise de 1 'anneau : "mâtre de la brousse et du village". Il attribue par ce geste tuus les pouvoirs (sur la brousse et le village) aux sefè (c'est à dire à la deuxième génération mythique).

C'est ainsi que le transfert des yapèrè s'achève par une sorte de mise hors jeu de la généalogie réelle.

Comment interprêter alors cette inversion gënéalogique ? Que sont les ancêtres de la deuxième gênëration pour qu'ils prennent ainsi le pas sur ceux de la première ? Lors de mes enquêtes, j'ai posé cette question aux initiês qui ont répondu que les sefè (deuxième génération) sont des esprits (gênies) supérieurs aux sefèzè (première génération). "Ce sont eux qui mettent la selle sur les possédés." "Ce sont eux les propriétaires du Nya et du village".

Mais, si les initiés accordent, comme il semble bien qu'ils l'affirment, une telle importance aux ancêtres de la deuxième génération, ce n'est pas, soulignons-le, en raison d'une vague croyance aux "génies rouges" ou génies tutélaires. I1 nous faut au contraire considérer avec les initiés le principe de ce renversement sefè / sefèzè en fonction d'une conception rigoureuse qu'ils se font de la création et de 1 'ordre cosmique de $1^{\prime}$ univers. Examinons à cet égard un récit de de la naissance des sefè.

"tyèzèrè (la vieille petite femme) ëtait en grossesse en brousse 
et n'arrivait pas à enfanter. nyglèrè (vieux petit commencement), son frère, interrogea la châ̂ne (chyg) qui lui fit savoir que cette difficulté annonçait une naissance de jumeaux qui leur seraient nettement supérieurs. De fait, tyèzèrè mit au monde des jumeaux anormalement constitués. Nés après terme, ils étaient soudés à un tronc unique et avaient le corps tuméfié par la lèpre. D'autre part, ils étaient dotès d'une intelligence éblouissante. Très vite, nanya, le jumeau (1itt. homme venu), conçut un plan pour se séparer de ses parents : il fit le mort et dit à sa soeur, pamanygh (1itt. action de bien faire venir) de le pleurer, ce qu'elle fit. "Je ne desirais pas partir, dit-elle, et tu me fait savoir à présent que nous partons, ils vont t'enterrer mort et ils vont m'enterrer vivante." Confus, nyglèrè se décida quand même à les enterrer en brousse (les jumeaux sont inextricablement reliès à un tronc unique). Mais à peine eut-il commencè à creuser une tombe que 1 'éclair de klè (klè yi ringè) l'aveugla. Les jumeaux en profitèrent pour s'enfuir et $s^{\prime}$ installer dans un bois touffu de la brousse (futur bois sacrê!" (18).

Ce drame gênéalogique, comne il ressort bien de la structure même de ce récit, peut être considéré, à bien des égards, comme une clé de voûte de toute l'histoire des générations de la brousse (dont se réclame $I^{\prime}$ humanité minyanka).

D'autre part, les deux yapèrè posés successivement en haut de la jarre du Nya représentent ces deux premières générations d'ancêtres (signes 1 et 2) s'opposant dans de nombreux récits. Les deux yapèrè portent le nom des ancêtres :

$$
\begin{array}{lc}
\text { sefèzè } & \begin{array}{c}
\text { sefè } \\
\text { "premiers ancêtres" }
\end{array} \\
\text { - première génération } & \text { - deuxième génération } \\
\text { - nyglèrè et tyèzèrè } & \text { - nanya et pamany̧̧h } \\
\text { - couple de jumeaux mixtes } & \text { - couple de jumeaux mixtes } \\
\text { - nés au cie1 } & \text { - nés sur terre } \\
\text { avant terme } & \text { après terme } \\
\text { - ancêtres décepteurs de } & \text { - ancêtres médiateurs entre } \\
1 \text { 'humanité } & 1 \text { 'humanité et klè (Dieu) }
\end{array}
$$

La disposition des yapèrè renvoit ainsi à un axe généalogique fondé sur le principe de la gëmel1ité. Bien connus chez les Bambara

(18) Il s'agit d'un récit que les initiês incorporent volontiers dans un cycle de contes et de mythes où les jumeaux prennent toujours le pas sur la génération de leurs parents, soit qu'ils les mettent à mort, soit qu'ils s'en sẻparent. 
Contribution à l'êtude des autels sacrificiels du Nya

sous le nom de pemba et mouso koroni (19), nyqzèrè et tyèzèrè forment ici le premier couple de jumeaux décepteurs de I'humanité. Nés au ciel avant terme, ce sont des êtres agités, instables, se livrant pendant une longue période mythique à la sorcellerie. Mais, alors que pemba et mousc koroni demeurent chez les Bambara sans descendance, nys zèrè et tyèzèrè donnent ici naissance à une paire de jumeaux mixtes (nanya et pamanygh) aux propriêtés monstrueuses (jumeaux lêpreux, soudés à un corps unique, etc...). Mais, il est clair en même temps que 1a pensée minyanka offre ici une solution originale pour accorder à tyèzèrè une descendance qui lui est partout ailleurs refusée (chez les Bambara elle reste stérile, chez les Dogon, elle meurt enceinte). Cette solution implique dans les récits que la mère enfante au terme d'une gestation lente et pénible une paire de jumeaux monstrueux qui se sêparent d'elle à leur naissance. Tout se passe en effet comme si la difformité des jumeaux était le prix payé par la pensée mythique pour lui accorder ici une descendance (d'ancêtres) dont elle est partout ailleurs dêpourvue. Mais, en même temps, cette pensée confère à la difformitê des jumeaux une signification positive : ils incarnent dans tous les rites des modes de médiation entre klè (Dieu) et les hommes.

Les jumeaux présentent trois types de déficiences organiques auxquelles on confère un pouvoir positif :

-- nês avec un retard considérable, les jumeaux figurent comme le produit d'une gestation bénéfique. Une naissance après terme est toujours bienvenue chez les Minyanka. Elle rappelle la naissance des premiers jumeaux de tyèzèrè. Le garçon portera le nom de nanya, la fille le nom de pamanygh. On prête aux enfants nés après terme une grande lucidité et une grande fermeté dans les décisions. On dira à la naissance du garçon : "ce sera un grand chef de terre". -- soudés à un tronc unique, les jumeaux témoignent d'un état de perfection ontologique - une sorte d'union indissoluble - état que leurs parents ont définitivement perdu depuis une vive querelle au ciel (ceux-ci errent ensuite sur terre en ne se retrouvant pas ou en ne se

(19) Les Minyanka ont adopté depuis longtemps la langue bambara et désignent très souvent le couple générateur de l'humanité, ny̧̨èrè et tyelèrè, par pemba et mouso koroni (en bambara). 
reconnaissant plus).

-- le corps tuméfié par la lèpre, ils témoignent d'une intelligence supérieure. C'est ainsi que, par extension, on dira du Nya qu'il a le pouvoir du lépreux. On clame à sa sortie :

"Nya gauche tu t'es levé

lépreux qui ne peut fabriquer une jarre

lépreux qui peut briser une jarre

les sorcières se sont encourues devant toi en jetant leurs pagnes Nya gauche tu t'es levé"

Or on sait que les jumeaux sont les propriétaires du Nya (nya kotigi). En outre, ils sont censẻs être invulnérables et jouent de ce fait un rôle dëfensif dans tous les rites d'antisorcellerie.

Mais il y a davantage, car la scène de la rupture des deux premières générations $n^{\prime} a$ de sens que si elle provoque en chaîne une sêrie d'autres ruptures généalogiques. tyèzèrè met en effet au monde d'autres enfants difformes (nains aux pieds à 1 'envers, unijambistes, sourds, etc...) qui occupent successivement des portions de brousse (colline, bois, mare, etc...) en se séparant d'elle. C'est ainsi qu'errante, tyèzèrè laisse derrière elle une brousse (mythique) qui se divise en autant de segments qu'elle y abandonne d'enfants monstrueux (les futurs gênies de lieu de la brousse).

Cette longue "dêrive gênéalogique" prend fin par une réconciliation inattendue entre tyèzèrè et ses premiers jumeaux. La réconciliation a lieu précisément au moment où elle retrouve son frère et tente de fonder avec lui un premier village :

"... affamés et errant sur des pistes où les avait êgarês la hyène, nyglèrè et tyèzèrè débouchent sur une colline rouge (ponyi kè). Trouvant la terre riche, ny̧lèrè décide d'y construire un premier village. Mais à peine a-t-il défriché la terre que surgissent d'un bois tout proche leurs premiers jumeaux (nanya et pamany̧h). nyglèrè les reconnât et leur adresse cette supplique : su bi nyanama woolo, nyanama bi su woolo (bambara)

"un mort peut accoucher d'un vivant, un vivant peut accoucher d'un mort".

La suite du récit indique clairement comment les jumeaux viennent en aide à la génération de leurs propres parents en exigeant d'eux un sacrifice sanglant au bois sacré.

Tel est, dans ces grandes 1ignes, 1 'épilogue d'un récit qui consacre la fin d'une longue période mythique. C'est un sacrifice au bois sacré - futur kachin kan - qui atténue la rupture des deux premières 
Contribution à l'étude des autels sacrificiels du Nya

générations d'ancêtres. Le récit les conjoint à l'aide d'un pacte sacririficiel qui leur assigne des emplacements différents :

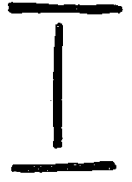

signe du ponyi kè

"colline rouge"
-- les sefètè (première gënẻration) occupent la "colline rouge", ponyi kè (20), lieu de fondation d'un premier village. -- les sefè (deuxième génération) occupent le "bois sacrē", kachin kan, d'où ils protègent la "colline rouge" et le village des

"premiers ancêtres".

Ajoutons que le sacrifice qui met fin à la rupture des deux premières gênérations d'ancêtres impose au moment de leur rencontre sur la "colline rouge". une sorte de renversement de leur position dans 1 'axe généalogique : les ancêtres de la première génération s'effacent devant ceux de la deuxième en leur adressant la supplique sacrificielle ("nous sommes.morts... vous êtes vivants").

Tout ceci justifie, aux yeux des initiês, la prescription rituelle qui recommande (mais pas toujours) d'inverser en haut de la jarre du Nya la position des deux yapèrè qui représentent les deux premières générations mythiques, le yapèrè des sefè (deuxième génération) devant obligatoirement coiffer celui des sefèlè (première gënération). La marque significative de cette inversion est, à n'en plus douter, une attitude ambivalente à l'égard des ancêtres de la première génération mythique.

Certes, les sefèzè coopèrent à l'ouvre de kỉè - au moment de la naissance des enfants, par exemple (voir p. 120)- mais ils sont toujours susceptibles, pense-t-on, de compromettre la création divine (comme ils l'ont fait à l'origine de l'humanité). C'est ainsi qu'on les tient pour responsables de ce que le Nya peut, en certaines circonstances, accepter le sacrifice des sorciers (le yapèrè des "premiers ancêtres" attirant nuitamment les sorciers vers le Nya).

Tout ceci justifie la position dominante du yapèrè des sefè, ancêtres de la deuxième génération, en haut de la troisième jarre du Nya.

(20) Pour se concilier les "premiers ancêtres", chaque chef de terre leur offre annuellement un sacrifice sur la "colline rouge". Mais ce sacrifice ne revêt pas l'importance de ceux offerts au "bois sacré", siège de la deuxième génêration mythique. 
L'ordre de disposition des yaperè est ainsi fonction d'une restauration généalogique du monde qui trouve son principe d'explication dans les mythes. Mais, alors que le pouvoir mortifère de la "vieille petite feme" est neutralisé dans toute I'aire mandé par les jumeaux de 1 'eau (Faro ou Nommo), ce sont ici les jumeaux difformes issus d'elle qui neutralisent son pouvoir de mère.

Ceci dit, nous allons voir à présent comment, au cours de la libation du sang de la victime, le yapèrè des sefè apparâ̂t comme un des agents les plus puissants de la médiation sacrificielle.

C. Libation du sang sacrificiel sur les yapèrè.

Célébré dans l'enclos du Nya après que les 266 yapèrè aient été rangés, selon un ordre rigoureux, dans les trois jarres sacrificielles, le rite de libation a pour but de rafraîchir les yapèrè avec le sang des victimes.

On ne peut manquer d'être frappë ici par l'extrême minutie du rituel en ce qui concerne la mise à mort du chien. Le rite se décompose en deux séquences bien distinctes :

-- les initiés assomment le chien en dehors de l'enclos en ramenant les pattes de 1 'animal le long de son échine, puis en le projetant violemment à terre (thêoriquement trois fois).

-- à 1'intérieur de l'encios, le sacrificateur égorge un petit poulet noir en énonçant une prière à caractère propitiatoire. On reconnâ̂t ici, comme partout ailleurs au Mandé, le rôle d'un petit sacrifice prêcêdant un sacrifice majeur. Le poulet une fois êgorgé, le sacrificateur suspend le chien au-dessus de la première jarre et, aiđé par quelques initiés, il ouvre unẹ large brèche de part et d'autre de la gorge du chien. Le couteau sacrificiel, dit soni muru, est de ce fait le premier yapèrè à être imprégné du sang de la victime. Après quoi, le sacrificateur fait tournoyer le chien en un mouvement qui épouse la courbe de la jarre, puis en le ramenant en fin de boucle vers le centre de celle-ci. Il répēte cette opération trois ou quatre fois jusqu'à épuisement du sang. Ainsi veille-t-il à répartir des parts de sang sur l'ensemble des yapèrè.

Au cours d'une récente enquête, je m'en serais tenu au constat 
Contribution à l'étude des autels sacrificiels du Nya

que tous les yapèrè ont été imprégnés du sang de la victime si mes informateurs n'avaient retenu mon attention sur une prescription élémentaire qui recommande de ne pas verser du sang sur le yapèrè des sefè (signe 2). Cet interdit qui consiste pour le sacrificateur à suspendre le geste de libation au-dessus d'un seul yapèrè - dans un ensemble de 266 éléments - tient au fait que ce yopèrè est l'habitacle de 1a puissance des jumeaux du Nya. Il occupe, nous I'avons vu, le haut de la troisième jarre. Voici comment les initiés interprètent cette phase du rite. "Après avoir versê du sang sur tous les yopèrè du Nya - à l'exception du yapèrè des jumeaux - nous avons 1 'habitude de quitter 1 'enclos... Au retour, que voyons-nous ? Les jumeaux sont descendus au fond de la jarre, puis ils sont remontés à la surface en aspirant du sang sur tous les yapèrè (21). En agissant ainsi, ils ont converti en haut de la jarre le sang lourd du chien en un sang léger (føgh fqghø) et l'ont ensuite renvoyê au chien avec le nyama, ou force vitale, du Nya."

Voilà dans sa simplicité l'interprétation qui m'a été faite. Elle indique clairement que la circulation du sang est placêe ici sous le contrôle du yapèrè des jumeaux (signe 2). Cette circulation sert à éliminer l'impureté du chien, ce qui est compréhensible chez les Minyanka où, comme partout ailleurs dans le Mandé, cet animal est censé porter toutes les impuretés du monde (le chien se nomne pun, avec un accent de rejet et de mépris).

I1 y a ainsi dans tout sacrifice au Nya une consécration de la victime, c'est à dire une sorte de transformation de son sang, du domaine "profane" (lourd) dans le domaine "sacré" (léger). C'est pourquoi les grands initiés font êtat de deux sortes de sangs dans la jarre :

-- le sang lourd du chien, versé par le sacrificateur sur tous les yapèrè, à l'exception de celui des jumeaux ;

- le sang léger du Nya, converti, à partir du sang lourd du chien, par I'action purifiante du yapèrè des jumeaux (ce yapèrè contient, rappelons-le, deux morceaux d'or, symboles de la pureté intangible de kZè).

(21) Les initiés n'hésitent pas à confondre l'objet (le yapèrè) et ce dont il est le réceptacle (1a puissance des jumeaux). 


\section{Philippe Jespers}

Ajoutons que ce sang léger, à peine perceptible, devient le vêhicule de la force vitale du Nya, le nyama, qui est, par le canal du sang, "divisible et transmissible", selon le schéma suivant :

yopèrè des jumeaux

$$
\begin{gathered}
\text { sang lourd } \\
\text { du chien } \\
\text { (visible) } \\
\text { nyama impur } \\
\text { (ou incomplet) } \\
\text { du chien }
\end{gathered}
$$

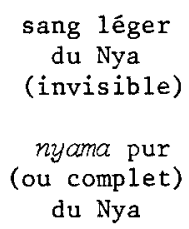

C'est ainsi que le nyama, qui constitue ici ce que $l^{\prime}$ 'on peut appeler la "force vitale du Nya" - c'est à dire la force contenue dans les 266 autels (ou signes de la création divine) - est renvoyé, par le canal d'un sang pur et léger, dans le corps de la victime.

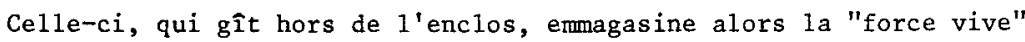
du Nya.

Ainsi, l'unité profonde du système sacrificiel tient à une filiation réelle des sacrifiants aux 266 yapèrè, supports des forces généalogiques de l'univers. Elle s'effectue à 1'aide d'un procédê qui consiste à renforcer les liens de filiation avec le Nya - les premiers yapèrè, rappelons-le, sont dits "ancêtres" - par 1'intermêdiaire d'une victime sacrifiée sur les autels.

v. Note sur la mort du chien du wara et du propriétaire du wara.

"L'enquête n'a pas rêvélê, comme chez les Dogon, le thème d'un héros mythique dont le sacrifice serait perpétuellement réactualisê par des rites sacrificiels"(22). Elle n'a jamais révélé, par exemple, de mise à mort $d^{\prime}$ un ancêtre consacrée par l'intention sacrificielle de Dieu (klè). Apparemment, tous les signes graphiques du Nya, de même que les nombreux récits et commentaires que nous avons suscités chez les Minyanka, font l'économie d'une telle figure.

(22) Danielle Jonckers, Contribution à l'étude du sacrifice chez les Minyanka. 
Contribution à l'étude des autels sacrificiels du Nya

Dès lors nous avons été amenés à laisser provisoirement cette question pour nous pencher sur un cycle de contes populaires qui relatent, sous une forme vivante, I'affrontement des chasseurs avec les wara, les animaux griffus, ou les siken chyen, les "petits hommes de la brousse". Ces récits ont tous pour but de rappeler les évènements remarquables qui se sont produits à l'origine entre le chasseur et le wara, et qui ont abouti à l'intégration des yapèrè au village. Référons-nous à cet égard à un récit de mise à mort des warablew (litt. fauves rouges), les "singes rouges" (Cercopithécus), anciens propriêtaires des sacs du Nya, c'est à dire propriétaires avant les hommes :

"un jour, un chasseur accompagné de son chien surprend un groupe de warablew (singes rouges) en train de voler des arachides dans un champ situé à quelques lieues du village. Très vite le chien met les singes en fuite, à l'exception de l'un d'eux qui, ployé sous la charge de trois sacs, ne peut éviter le chien qui l'égorge. De retour au village, le chien livre les secrets de la composition des sacs et meurt sur le coup. C'est pourquoi, ajoute le narrateur, on égorge aujourd'hui des chiens au Nya."

On voit par ce récit que la connaissance du Nya procède d'une mise à mort violente de ses anciens propriétaires de brousse. Or il s'agit ici d'une loi générale de tous les récits qui évoquent le transit des yapèrè de la brousse au village. C'est ainsi que l'appropriation du Nya passe par la mort des "singes rouges", celle du nankon (sociêté des chasseurs) par la mort de 1'hyène, celle du satggho (société des agriculteurs) par la mort de la même hyène, celle du tyè (yapèrè de sorcellerie) par la mort du lièvre, celle du manyan (mère du Nya) par le meurtre d'une femme muette de brousse, celle du chirar chira (yapèrè de la mare) par la mort du lamentin (ou par l'amputation de ses bras selon une autre version), celle du pqpqchy (jarre des Bobo) par le meurtre d'une femme bobo (de l'ethnie voisine des Minyanka) etc...

Nous ne pouvons nous étendre davantage dans cette énumération rapide qui ne vise ici qu'à tracer les grandes lignes du phénomène de capture des yapèrè. Mais, d'ores et déjà, il apparaît clairement qu'à l'origine toutes les grandes puissances de la brousse (les yopèrè) étaient aux mains des wara, les animaux griffus, ou des siken chyen, les "petits hommes de brousse" ou même des hommes déchus en animaux, comme les warablew, "singes rouges", qui les conservaient généralement dans des placentas d'antilope (ainsi, les prestigieux yapèrè du Nya). 
Leur classification fait place ici - un peu comme on 1'a fréquemment souligné pour le totémisme - à une extraordinaire généalogie d'animaux ou de monstres qui nécessiterait, pour être pleinement comprise, 1a mise en oeuvre d'un grand nombre de rêcits.

Toute aussi éclairante à cet égard est la planchette des signes du Nya. Elle est une récapitulation exhaustive de tous les segments généalogiques issus des sefêlè, les premiers ancêtres, lesquels, rappelons-nous, figurent en tête de la planchette. Viennent ensuite les jumeaux monstrueux, les wara et les "petits hommes de brousse" en des séries de signes superposés (dits "chemins") où figurent également au complet les prestigieux yapèrè qu'ils ont reçu de Dieu pour se protéger dans leurs territoires. Sur 86 signes graphiques, 50 représentent ces yopèrè ou leurs lieux d'implantation originaires en brousse.

Si la pensée accorde, comme il semble bien que les rêcits l'indiquent, une telle importance aux problèmes des distances, des êtres intermédiaires (wara et petits hommes) et des opêrations effectuées sur un espace de brousse (prise ou détournement d'un yapèrè), ce n'est pas en raison d'un caprice pervers d'une intelligence vouée à explorer des lieux de brousse inexplicablement conformes à la nature des yapèrè. D'innombrables récits montreraient que 1 'intrusion dans ces espaces (bois, termitière, colline etc...) - situés souvent à la limite du territoire - a toujours pour enjeu les yapèrè détenus par les wara ou les "petits hommes".

Tout ceci peut aider, sans doute, à mieux comprendre pourquoi le chien est devenu la victime sacrificielle du Nya (et de bien d'autres institutions religieuses minyanka). Animal au flair infaillible, assurant en quelque sorte le relais entre le monde domestique et le monde sauvage, c'est toujours lui qui accompagne le chasseur au moment de la conquête des yapèrè. Que certains récits nous rêvèlent que le chien meurt aussi pour avoir égorgé les warablew ("singes rouges") et avoir livré les secrets de la composition du Nya, qu'ils établissent par là un lien explicite entre la mort du $w a r a-$ nom que 1 'on donne fréquemment au Nya - et 1a mort du chien, voici qui n'est pas sans éclairer, croyons-nous, un des aspects de l'acte sacrificiel.

Mais, exalté dans de nombreux récits, l'acte de cette violence mythique ne s'achève pas pour autant avec la mort de 1 'animal domestique. Il entraîne également dans son propre mouvement la mort de 
- Contribution à l'étude des autels sacrificiels du Nya

celui même qui s'est approprié le Nya à titre de nya tigi, "propriétaire du Nya" (ce qui constitue en soi une des sept classes initiatiques de la sociétê). Et aujourd'hui encore, lorsqu'un candidat à une telle puissance se rend auprès d'un "mâ̂tre de la composition" (dans un autre village), celui-ci le prévient qu'un acte de cette nature le fera passer à trépas. "Le Nya, dit-on, lui mettra le mors et il périra" (quelques fragments de son corps seront alors intégrés dans la composition des sacs).

C'est donc, si l'on prend un peu de recul, une sorte d'échange entre un animal et un homme, projetés tour à tour au-delà des limites de la vie. Mais alors que le wara meurt une fois pour toutes au moment de I'intégration du premier Nya au village, la puissance du Nya est, par contre, à ce point redoutable qu'elle réclame à chacune de ses reconstitutions au village la mort de ses nouveaux propriétaires (seIon une chaîne territoriale de filiation qui passe de village en village).

Tel est le pacte de meurtre qui ponctue cette châ̂ne et qui est au fondement de 1'institution. Quant aux descendants d'un ancêtre fondateur de Nya (de village ou de quartier), ils se contenteront dans la suite de sacrifier des chiens dans 1 'enclos du nya tun, à intervalles réguliers et en invoquant dans leurs prières le nom de l'ancêtre.

Ainsi pouvons-nous provisoirement articuler pour le Nya l'acte de mise à mort sacrificiel du chien (sans avoir pu pour autant dégager la transformation de champ que cela implique) et l'acte de mise à mort originel d'un animal mythique, le warablew. Le sacrifice ren-

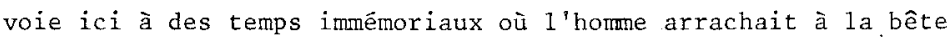
mythịque de la brousse les yapèrè. 